\title{
QA-Driven Zero-shot Slot Filling with Weak Supervision Pretraining
}

\author{
Xinya Du* \\ Cornell University \\ xdu@cs.cornell.edu
}

\author{
Dian Yu* \\ University of California, Davis \\ dianyu@ucdavis.edu
}

\author{
Luheng He \\ Google Research \\ luheng@google.com \\ Panupong Pasupat \\ Google Research \\ ppasupat@google.com
}

Qi Li

Google Assistant qilqil@google.com

\author{
Yuan Zhang \\ Google Research \\ zhangyua@google.com
}

\begin{abstract}
Slot-filling is an essential component for building task-oriented dialog systems. In this work, we focus on the zero-shot slot-filling problem, where the model needs to predict slots and their values, given utterances from new domains without training on the target domain. Prior methods directly encode slot descriptions to generalize to unseen slot types. However, raw slot descriptions are often ambiguous and do not encode enough semantic information, limiting the models' zero-shot capability. To address this problem, we introduce QA-driven slot filling (QASF), which extracts slot-filler spans from utterances with a span-based QA model. We use a linguistically motivated questioning strategy to turn descriptions into questions, allowing the model to generalize to unseen slot types. Moreover, our QASF model can benefit from weak supervision signals from QA pairs synthetically generated from unlabeled conversations. Our full system substantially outperforms baselines by over $5 \%$ on the SNIPS benchmark.
\end{abstract}

\section{Introduction}

Automatic slot filling, which extracts task-specific slot fillers (e.g. flight date, cuisine) from user utterances, is an essential component to spoken language understanding (Bapna et al., 2017). As shown in Figure 1, the model predicts the slot filler "Joe A. Pass" for the slot type "artist" given an input utterance. However, fully supervised slot filling models (Young, 2002; Goo et al., 2018) require labeled training data for each type of slot (Shah et al., 2019). It is even more of a problem for dataintensive models (Mesnil et al., 2014). This makes the development of new domains in these systems a challenging and resource-intensive task.

This has motivated studies in cross-domain zeroshot learning for the slot-filling task (ZSSF), where

\footnotetext{
Work done during internship at Google Research.
}

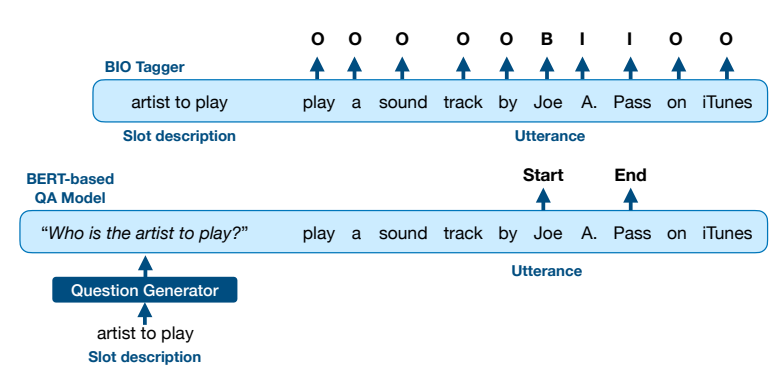

Figure 1: Comparison between two slot-filling frameworks: BIO tagging based model (upper) and our QAbased model (below).

the goal is to achieve good slot-filling performance on new domains without requiring additional training data. Previous work (Bapna et al. (2017); Shah et al. (2019)) often uses a sequence tagging approach (similar to the upper image in Figure 1 in a high-level way). To achieve zero-shot domain transfer, they directly encode raw slot descriptions or names, such as "playlist", "music item", to enable models to generalize to slot types unseen at training time. However, slot descriptions are often ambiguous and typically do not encode enough semantic information by themselves.

Instead of directly encoding slot descriptions and examples, we introduce a QA-driven slot filling framework (QASF) (Figure 1). Inspired by the recent success of QA-driven approaches (McCann et al., 2018; Logeswaran et al., 2019; Gao et al., 2019; Li et al., 2020; Namazifar et al., 2020), we tackle the slot-filling problem as a reading comprehension task, where each slot type (e.g. "artist") is associated with a natural language question (e.g. "Who is the artist to play?"). A span-based reading comprehension model is then used to extract a slot filler span from the utterance by answering the question. ${ }^{1}$ In this work, we use a linguisti-

\footnotetext{
${ }^{1}$ It can been seen as an extension of the QA-driven meaning representations (He et al., 2015; Michael et al., 2017), where
} 
cally motivated question generation strategy for converting slot descriptions and example values into natural questions, followed by a BERT-based QA model for extracting slot fillers by answering questions. As shown in our experiments, this QAdriven method is better at exploiting the semantic information encoded in the questions, therefore it generalizes better to new domains without any additional fine-tuning, as long as the questions are meaningful enough. To the best of our knowledge, we are the first to leverage weakly supervised synthetic QA pairs extracted from unlabeled conversations for a second-stage pretraining. Drawing insights from Mintz et al. (2009), we create a weakly supervised QA dataset from unlabeled conversations and an associated ontology. The synthetic QA pairs are constructed by matching unlabeled utterances against possible slot values in the ontology. This provides a general and cost-effective way to improve QA-based slot filling performance with easily obtainable data.

Experimental results show that (1) our QASF model significantly outperforms previous zeroshot systems on SNIPS (Coucke et al., 2018) and TOP (Gupta et al., 2018); (2) encoding natural questions help models better leverage weakly supervised signals in the pretraining phase, compared to encoding raw descriptions.

\section{Task Definition}

Given an input utterance $u$, a slot filling model extracts a set of (slot type, span) pairs $\left(s_{i}, a_{i}\right), i=$ $1, \ldots, k$ where $s_{i}$ comes from a fixed set of slot types $\mathcal{S}$, and each $a_{i}=(j, k), 1 \leq j<k \leq$ $|u|$ is a span in $u$. Each slot type is accompanied with a short textual description that describes its semantic meaning (Table 1). We also assume that a small amount of example slot values are given, following Shah et al. (2019).

Our goal is to build a slot filling model that performs well on a new target domain with unseen slot types. Our training data consists of utterances from $N$ source domains $\mathcal{D}_{1}, \mathcal{D}_{2}, \ldots, \mathcal{D}_{N}$. Each domain $\mathcal{D}_{i}$ is associated with a set of predefined slot types $S_{i}$. At test time, utterances are drawn from a new domain $\mathcal{D}_{N+1}$. The new domain contains both seen and unseen slot types from the source domain. For example, in the SNIPS dataset (Coucke et al., 2018), domains "GetWeather" and "BookRestaurant" both

predicate-argument structures are represented as QA pairs. have a slot type called "city", while "condition_temperature" only appears in the "GetWeather" domain.

\section{Methodology}

In this section, we describe our framework for Question Answer-driven Slot Filling (QASF). The framework consists of (1) a question generation strategy that turns slot descriptions into natural language questions based on linguistic rules; (2) a generic span-extraction-based question answering model; (3) an intermediate pretraining stage with generated synthetic QA pairs from unlabeled conversations, which is before task-specific training.

\subsection{Question Generation Strategy}

To benefit from both language model pretraining and QA supervision, we design a question generation strategy to turn slot descriptions into natural questions. During this process, a considerable amount of knowledge and semantic information is encoded (Heilman, 2011). A generated question consists of a WH word and a normalized slot description following the template below:

$$
\text { WH_word is slot_description? }
$$

Generating WH_word We draw insights from the literature on automatic question generation. Heilman and Smith (2010) propose to use linguistically motivated rules. In their more general case of question generation from the sentence, answer phrases can be noun phrases (NP), prepositional phrases (PP), or subordinate clauses (SBAR). Complicated rules are designed with help from superTagger (Ciaramita and Altun, 2006).

For our spoken language understanding (SLU) tasks, slot fillers are mostly noun phrases ${ }^{2}$. Therefore, we design a simpler set of conditions based on named entity types and part-of-speech (POS) tags. For each slot type, we sample 10 (utterance, slot value) examples from the validation set. Then we run a NER and a POS tagging model ${ }^{3}$ to obtain entity types and POS tags for each of the sampled answer spans. Finally, we select $\mathrm{WH}$ _word based on a set of rules described in Table 6 in Appendix.

Generating slot_description Instead of directly adding a raw description phrase in the question template, we normalize the phrase with the

\footnotetext{
${ }^{2}$ around $90 \%$ cases in the SNIPS dataset.

${ }^{3}$ Provided by spaCy: https: / / spacy.io/
} 


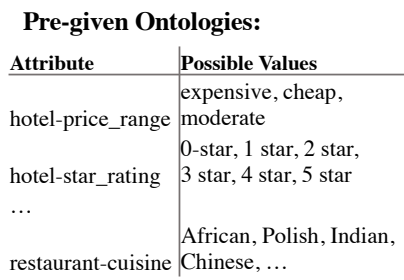

Unlabeled Conversation Example:

USER: I am looking for a place to stay in the north of the city. I would prefer a 4-star hotel please.

SYS: There are several guesthouses available. Do you have a price reference?

USER: The restaurant should be in the moderate price range.
USER: I am looking for a place to stay in the north of the city. I would prefer a 4 star [star_rating] hotel please.

String matching

SYS: There are several guesthouses available. Do you have a price reference?

USER: The restaurant price should be in the moderate [price_range].

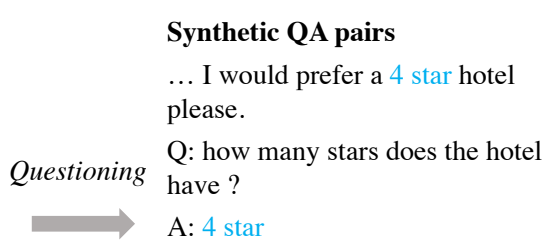

The restaurant price should be in the moderate.

$\mathrm{Q}$ : How is the price?

A: moderate

Figure 2: Obtaining weakly supervised synthetic QA pairs for pretraining. Given an ontology and unlabeled utterances, we generate synthetic QA pairs with weak supervision by matching values of slots against the utterances.

\begin{tabular}{l|ll}
\hline Slot & Raw Description & Our Question \\
\hline playlist_owner & owner & who's the owner? \\
\hline object_select & object select & which object to select? \\
\hline best_rating & points in total & how many points in total? \\
\hline num_book_people & $\begin{array}{l}\text { number of people } \\
\text { for booking }\end{array}$ & $\begin{array}{l}\text { how many people } \\
\text { for booking? }\end{array}$ \\
\hline
\end{tabular}

Table 1: Examples of generated questions.

following simple rule: If the description is of the format " $A$ of $B$ ", where both $A$ and $B$ are noun phrases (NP), we only keep $B$ in the phrase if the WH_word is "How long" or "How many". Examples of generated questions for corresponding slots are presented in Table 1. Compared to slot descriptions, our questions are more precise and can encode more semantic information.

\subsection{Question Answering Model}

We use BERT (Devlin et al., 2019) as our base model for jointly encoding the question and utterance. Input sequences for the model share a standard BERT-style format: [CLS] < question > [SEP] $<$ utterance $>[S E P]$, where [CLS] is BERT's special classification token and [SEP] is the special token to denote separation. Let $\mathbf{e}_{1: M}$ be the token-level output representation from the BERT encoder,

$$
\mathbf{e}_{1}, \mathbf{e}_{2}, \ldots, \mathbf{e}_{M}=\operatorname{BERT}\left(x_{1}, x_{2}, \ldots, x_{M}\right)
$$

where $x_{1: M}$ are the input tokens.

Then the model predicts answer spans with two binary classifiers on top of the BERT outputs $\mathbf{e}_{1: M}$. The two classifiers are trained to predict whether each token is the start or the end of an answer span, respectively,

$$
\begin{aligned}
& P_{\mathrm{s}}(i \mid i \in 1 \ldots M)=\operatorname{softmax}\left(\mathbf{e}_{i} \mathbf{W}_{\mathrm{s}}\right) \\
& P_{\mathrm{e}}(i \mid i \in 1 \ldots M)=\operatorname{softmax}\left(\mathbf{e}_{i} \mathbf{W}_{\mathrm{e}}\right)
\end{aligned}
$$

For negative examples, where a question has no answer spans in the utterance, we map the start and end token both to the [CLS] token. During training, we minimize the negative log-likelihood loss. All parameters are updated. During inference, predicting slot filler spans is more complex because there could be several or no spans to be extracted for each slot type. We first enumerate all possible spans and only keeping spans/answers satisfying certain constraints (Appendix Section B) as fillers.

\subsection{Pretraining with Weak Supervision}

Pretrained masked language models do not have the capability of question answering before being fine-tuned on task-specific data. We hypothesize that adding a pretraining step with synthetic QA pairs before fine-tuning can contribute to models' understanding of interactions between question and utterance. For example, improvements have been reported by QAMR (He et al., 2020) on SRL and textual entailment (TE). Previous researches (Wu et al., 2020; Gao et al., 2020) have used crowdsourced QA pairs, but typically the improvement margin is not significant (Wu et al., 2020) when the task-specific data is in a different domain (SQuAD v.s. newswire). Therefore we introduce a method of collecting relevant and distantly supervised QA pairs and investigate their influences in pretraining. More specifically, we draw insights from 
Mintz et al. (2009) for creating a weakly supervised dataset. Figure 2 illustrates the process. Given an ontology or database of slot types and all possible values for each slot type, we find all utterances containing those value strings in a large set of unlabeled conversations. For example, in Figure 2, for the "hotel_price_range" slot, there are three possible values "expensive", "cheap" and "moderate" in the ontology. We then form question-answerutterance triples using the question generation strategy proposed in Section 3.1.

To obtain the pre-defined ontology and unlabeled conversations, we use MultiWOZ 2.2 (Zang et al., 2020), which is an improved version of MultiWOZ (Budzianowski et al., 2018). We do not use annotations in the dataset such as the (changes of) states in the conversations and we treat each utterance independently. We remove slot types that exist in the task-specific training/test data (i.e., SNIPS and TOP) from the ontology and end up with 67,370 QA examples for pretraining.

\section{Experiments}

\subsection{Datasets and Baselines}

SNIPS (Coucke et al., 2018) is an SLU dataset consisting of crowdsourced user utterances with 39 slots across 7 domains - "AddToPlaylist" (ATP), "BookRestaurant" (BR), "GetWeather" (GW), "PlayMusic" (PM), "RateBook" (RB), "SearchCreativeWork" (SCW), "FindScreeningEvent" (FSE). It has around 2000 training instances per domain. The slot types of each domain do not overlap with each other. Following previous work (Shah et al., 2019; Liu et al., 2020), we use this dataset to evaluate zero-shot cross-domain transfer learning - train on all training instances from domains other than $D_{i}$, and test exclusively on $D_{i}$, for $i=1, \ldots, 7$. TOP (Gupta et al., 2018) is a task-oriented utterance parsing dataset. It is based on a hierarchical annotation scheme for annotating utterances with nested intents and slots. Each slot type also comes with a description. In our setup, we train on all seven domains of SNIPS as well as varying amounts of training data from the TOP training set $(0,20$, and 50 examples), and use the TOP test set as an out-of-distribution domain for evaluation. We report span-level F1 (micro-average).

We compare our method against a number of representative baselines. Concept Tagger (CT) (Bapna et al., 2017) is a slot-filling framework that directly uses original slot descriptions to general- ize to unseen slot types. Robust Zero-shot Tagger (RZT) (Shah et al., 2019) is an extension of $\mathrm{CT}$, which incorporates example values of slots to improve the robustness of the model's zero-shot capability. Coach (Liu et al., 2020) is a coarse-tofine model for slot-filling. It also encodes raw slot descriptions. We also include a Zero-Shot BERT Tagger (ZSBT) based on BERT (Devlin et al., 2019) as an additional baseline. ZSBT directly encodes raw slot descriptions and utterances and predicts a $\operatorname{tag}(\mathrm{B}, \mathrm{I}$, or $\mathrm{O})$ for each token in the utterance.

\subsection{Results and Analysis}

We report F-1 of the baselines and our model on each target domain test set of SNIPS as well as average F-1 across domains. All models are trained on the other six domains for each target domain. As shown in Table 2, our QA-driven slot filling framework (QASF) significantly outperforms all baselines in five of the seven domains, with slightly lower performance on BookRestaurant than ZSBT, and lower performance on FindScreeningEvent than Coach. The average F-1 of QASF is around 7\% higher than the prior published state-of-the-art Coach model, and about $2 \%$ higher than the Zero-shot BERT Tagger baseline. Adding the intermediate pre-training stage on weakly supervised data further improves performance on top of QASF in six of the seven domains except for AddToPlaylist. On average, adding pre-training improves over QASF by $2.9 \% \mathrm{~F}-1$. The zero-shot performance of all models are relatively worse on PlayMusic, RateBook and FindScreeningEvent. A more detailed discussion is in Appendix Section C.

Table 3 summarizes TOP test results: (1) In both the zero-shot and few-shot settings, our QASF outperforms ZSBT, with a bigger improvement on the zero-shot setting. (2) Pretraining on the weakly supervised QA pairs helps more in the zero-shot setting than in the few-shot setting, with a $20 \%$ relative improvement. This shows that QASF (w/ pretraining) is more robust to the domain shift when there is no target domain training data.

Impact of QG strategy and pretraining To understand the influence of question generation and impact of pretraining with synthetic QA pairs, we perform ablation studies of both components on the SNIPS dataset. The table below shows ablation results (F-1). "w/o QG" refers to a model trained with raw slot descriptions and utterances.

Firstly, the question generation strategy consis- 


\begin{tabular}{l|ccccccc|c}
\hline & ATP & BR & GW & PM & RB & SCW & FSE & Average F-1 \\
\hline CT (Bapna et al., 2017) & 38.82 & 27.54 & 46.45 & 32.86 & 14.54 & 39.79 & 13.83 & 30.55 \\
RZT (Shah et al., 2019) & 42.77 & 30.68 & 50.28 & 33.12 & 16.43 & 44.45 & 12.25 & 32.85 \\
Coach (Liu et al., 2020) & 50.90 & 34.01 & 50.47 & 32.01 & 22.06 & 46.65 & 25.63 & 37.39 \\
ZSBT $^{\text {BERT }}$ (our baseline) & 55.78 & $\mathbf{4 9 . 3 4}$ & 56.58 & 28.35 & 27.09 & 57.61 & 20.50 & 42.18 \\
\hline QASF $^{\text {BERT }}$ (ours) & $\mathbf{5 9 . 2 9}$ & 43.13 & 59.02 & 33.62 & 33.34 & 59.90 & 22.83 & 44.45 \\
w/ pre-training on WS & 57.57 & 48.75 & $\mathbf{6 1 . 2 7}^{*}$ & $\mathbf{3 8 . 5 4}^{*}$ & $\mathbf{3 6 . 5 1}^{* *}$ & $\mathbf{6 0 . 8 2}^{\mathbf{2 7 . 7 2}^{* *}}$ & 47.31 \\
\hline
\end{tabular}

Table 2: Experimental results (F-1) on SNIPS dataset. ${ }^{*}$ indicates statistical significance $(\mathrm{p}<0.05),{ }^{* *}: \mathrm{p}<0.01$.

\begin{tabular}{l|ccc}
\hline & Zero-shot & Few-shot (20) & Few-shot (50) \\
\hline Random NE & 1.34 & - & - \\
ZSBT & 8.82 & 37.60 & 42.73 \\
QASF (ours) & 10.27 & 36.86 & 46.49 \\
w/ pre-training on WS & 12.35 & 39.78 & 47.91 \\
\hline
\end{tabular}

Table 3: Evaluation results on TOP test. Models trained on SNIPS, and varying amount of utterances of TOP train - zero-shot, 20 -shot (1\%), 50-shot $(2.5 \%)$.

\begin{tabular}{ccc|ccc}
\hline \multicolumn{3}{c|}{ w/o pretraining } & \multicolumn{3}{c}{ w/ pretraining } \\
\hline QASF & w/o QG & $\Delta$ (F1) & QASF & w/o QG & $\Delta(\mathrm{F} 1)$ \\
44.45 & 41.97 & $+5.91 \%$ & 47.31 & 43.09 & $+9.79 \%$ \\
\hline
\end{tabular}

Table 4: Ablation Study

tently helps, with a $2.48 \%$ F-1 gain in "w/o pretraining" and a $4.22 \%$ F-1 gain in "w/ pretraining". Secondly, the pretrained representations from additional weakly supervised data improve F-1 by $2.86 \%$ in "w/ QG" and $1.12 \%$ in "w/o QG". More interestingly, the gain from the questioning strategy is larger when combined with the pretraining $(9.8 \%$ as compared to $5.9 \%$ ). This demonstrates that synthetic QA pairs are also helping with getting better QA-aware representations before fine-tuning on the task-specific data for slot-filling.

\subsection{Error Analysis}

We further conduct manual error analysis on the models' predictions on SNIPS. We find that there are several sources where the errors are from:

The variance between the source and target domains. Sometimes even slot types of the same name refer to different kinds of objects in different domains. For example, slot type "object_type" in the "RateBook" domain refers to object types like textbook, essay and novel; while in the "FindScreeningEvent", it refers to event types like movie times/schedules. In the two domains, they have the same raw descriptions. In the table below, we show the performance of models on utterances with "object_type" and "object_name" spans (according to gold annotations). We can see that the performances on these special slots are significantly

\begin{tabular}{c|ccc} 
& ZSBT & QASF & QASF (w/ pretraining) \\
\hline Averaged F-1 & 17.43 & 22.26 & 24.29 \\
\hline
\end{tabular}

lower than the general average on all the examples (40-50\%). But still, the questioning strategy helps improve the transferring of semantic information.

Plus, the variance in semantic meaning between slot types in SNIPS and TOPS is even larger. For slots like "location_modifier", "road_condition", there are no semantic similar slots in SNIPS or pretraining dataset, which results in low performance. Having more specific/detailed slot descriptions and use them in the question generation would help further (Brown et al., 2020; Du and Cardie, 2020).

Annotation artifacts of SNIPS dataset and sparsity of vocabulary for certain slot types. Our QASF framework does not perform well on the target domain "BookRestaurant", thus we take a close look at it. We find that there are only 25 possible values in total for slot restaurant_type, over $\mathbf{5 1 \%}$ of them are of a single token "restaurant" (Table below). A very simple approach (assigning type "restaurant_type" to all tokens "restaurant" can obtain decent performance). This does not happen for

\begin{tabular}{c|cccc}
\hline Slot Value & "restaurant" & "bar" & "pub" & "brasserie" \\
\hline Proportion & $51.81 \%$ & $7.26 \%$ & $6.60 \%$ & $6.23 \%$ \\
\hline
\end{tabular}

other slot types in BookRestaurant (e.g., cuisine, restaurant_name). The possible values are more diverse and the distribution is more balanced.

\section{Conclusion}

We propose a QA-driven method with weakly supervised pretraining for zero-shot slot filling. Our experimental results and analyses demonstrate the benefits of QA-formulation, especially in the setting with synthetic QA pairs pretraining.

\section{Acknowledgments}

We thank Kenton Lee and Emily Pitler, and anonymous reviewers for their constructive suggestions. 


\section{References}

Ankur Bapna, Gokhan Tür, Dilek Hakkani-Tür, and Larry Heck. 2017. Towards zero-shot frame semantic parsing for domain scaling. In Proc. Interspeech 2017, pages 2476-2480.

T. Brown, B. Mann, Nick Ryder, Melanie Subbiah, J. Kaplan, P. Dhariwal, Arvind Neelakantan, Pranav Shyam, Girish Sastry, Amanda Askell, Sandhini Agarwal, Ariel Herbert-Voss, G. Krüger, Tom Henighan, R. Child, Aditya Ramesh, D. Ziegler, Jeffrey Wu, Clemens Winter, Christopher Hesse, Mark Chen, E. Sigler, Mateusz Litwin, Scott Gray, Benjamin Chess, J. Clark, Christopher Berner, Sam McCandlish, A. Radford, Ilya Sutskever, and Dario Amodei. 2020. Language models are few-shot learners. ArXiv, abs/2005.14165.

Paweł Budzianowski, Tsung-Hsien Wen, Bo-Hsiang Tseng, Iñigo Casanueva, Stefan Ultes, Osman Ramadan, and Milica Gašić. 2018. MultiWOZ - a large-scale multi-domain Wizard-of-Oz dataset for task-oriented dialogue modelling. In Proceedings of the 2018 Conference on Empirical Methods in Natural Language Processing, pages 5016-5026, Brussels, Belgium. Association for Computational Linguistics.

Massimiliano Ciaramita and Yasemin Altun. 2006. Broad-coverage sense disambiguation and information extraction with a supersense sequence tagger. In Proceedings of the 2006 Conference on Empirical Methods in Natural Language Processing, pages 594-602.

Alice Coucke, Alaa Saade, Adrien Ball, Théodore Bluche, Alexandre Caulier, David Leroy, Clément Doumouro, Thibault Gisselbrecht, Francesco Caltagirone, Thibaut Lavril, et al. 2018. Snips voice platform: an embedded spoken language understanding system for private-by-design voice interfaces. arXiv preprint arXiv:1805.10190.

Jacob Devlin, Ming-Wei Chang, Kenton Lee, and Kristina Toutanova. 2019. BERT: Pre-training of deep bidirectional transformers for language understanding. In Proceedings of the 2019 Conference of the North American Chapter of the Association for Computational Linguistics: Human Language Technologies, Volume 1 (Long and Short Papers), pages 4171-4186, Minneapolis, Minnesota. Association for Computational Linguistics.

Xinya Du and Claire Cardie. 2020. Event extraction by answering (almost) natural questions. In Proceedings of the 2020 Conference on Empirical Methods in Natural Language Processing. Association for Computational Linguistics.

Shuyang Gao, Sanchit Agarwal, Di Jin, Tagyoung Chung, and Dilek Hakkani-Tur. 2020. From machine reading comprehension to dialogue state tracking: Bridging the gap. In Proceedings of the 2 nd
Workshop on Natural Language Processing for Conversational AI, pages 79-89, Online. Association for Computational Linguistics.

Shuyang Gao, Abhishek Sethi, Sanchit Agarwal, Tagyoung Chung, and Dilek Hakkani-Tur. 2019. Dialog state tracking: A neural reading comprehension approach. In Proceedings of the 20th Annual SIGdial Meeting on Discourse and Dialogue, pages 264-273, Stockholm, Sweden. Association for Computational Linguistics.

Chih-Wen Goo, Guang Gao, Yun-Kai Hsu, Chih-Li Huo, Tsung-Chieh Chen, Keng-Wei Hsu, and YunNung Chen. 2018. Slot-gated modeling for joint slot filling and intent prediction. In Proceedings of the 2018 Conference of the North American Chapter of the Association for Computational Linguistics: Human Language Technologies, Volume 2 (Short Papers), pages 753-757.

Sonal Gupta, Rushin Shah, Mrinal Mohit, Anuj Kumar, and Mike Lewis. 2018. Semantic parsing for task oriented dialog using hierarchical representations. In Proceedings of the 2018 Conference on Empirical Methods in Natural Language Processing, pages 2787-2792, Brussels, Belgium. Association for Computational Linguistics.

Hangfeng He, Qiang Ning, and Dan Roth. 2020. QuASE: Question-answer driven sentence encoding. In Proceedings of the 58th Annual Meeting of the Association for Computational Linguistics, pages 8743-8758, Online. Association for Computational Linguistics.

Luheng He, M. Lewis, and Luke Zettlemoyer. 2015. Question-answer driven semantic role labeling: Using natural language to annotate natural language. In EMNLP.

Michael Heilman. 2011. Automatic factual question generation from text. Ph.D. thesis, Ph. D. thesis, Carnegie Mellon University.

Michael Heilman and Noah A Smith. 2010. Good question! statistical ranking for question generation. In Human Language Technologies: The 2010 Annual Conference of the North American Chapter of the Association for Computational Linguistics, pages 609617.

Xiaoya Li, Jingrong Feng, Yuxian Meng, Qinghong Han, Fei Wu, and Jiwei Li. 2020. A unified MRC framework for named entity recognition. In Proceedings of the 58th Annual Meeting of the Association for Computational Linguistics, pages 58495859, Online. Association for Computational Linguistics.

Zihan Liu, Genta Indra Winata, Peng Xu, and Pascale Fung. 2020. Coach: A coarse-to-fine approach for cross-domain slot filling. In Proceedings of the 58th Annual Meeting of the Association for Computational Linguistics, pages 19-25, Online. Association for Computational Linguistics. 
Lajanugen Logeswaran, Ming-Wei Chang, Kenton Lee, Kristina Toutanova, Jacob Devlin, and Honglak Lee. 2019. Zero-shot entity linking by reading entity descriptions. In Proceedings of the 57th Annual Meeting of the Association for Computational Linguistics, pages 3449-3460, Florence, Italy. Association for Computational Linguistics.

Bryan McCann, Nitish Shirish Keskar, Caiming Xiong, and Richard Socher. 2018. The natural language decathlon: Multitask learning as question answering. arXiv preprint arXiv:1806.08730.

Grégoire Mesnil, Yann Dauphin, Kaisheng Yao, Yoshua Bengio, Li Deng, Dilek Hakkani-Tur, Xiaodong He, Larry Heck, Gokhan Tur, Dong Yu, et al. 2014. Using recurrent neural networks for slot filling in spoken language understanding. IEEE/ACM Transactions on Audio, Speech, and Language Processing, 23(3):530-539.

Julian Michael, Gabriel Stanovsky, Luheng He, I. Dagan, and Luke Zettlemoyer. 2017. Crowdsourcing question-answer meaning representations. ArXiv, abs/1711.05885:560-568.

Mike Mintz, Steven Bills, Rion Snow, and Daniel Jurafsky. 2009. Distant supervision for relation extraction without labeled data. In Proceedings of the Joint Conference of the 47th Annual Meeting of the ACL and the 4th International Joint Conference on Natural Language Processing of the AFNLP, pages 1003-1011, Suntec, Singapore. Association for Computational Linguistics.

Mahdi Namazifar, Alexandros Papangelis, Gokhan Tur, and Dilek Hakkani-Tür. 2020. Language model is all you need: Natural language understanding as question answering. arXiv preprint arXiv:2011.03023.

Feng Pan, Rutu Mulkar-Mehta, and Jerry R Hobbs. 2011. Annotating and learning event durations in text. Computational Linguistics, 37(4):727-752.

Darsh Shah, Raghav Gupta, Amir Fayazi, and Dilek Hakkani-Tur. 2019. Robust zero-shot cross-domain slot filling with example values. In Proceedings of the 57th Annual Meeting of the Association for Computational Linguistics, pages 5484-5490, Florence, Italy. Association for Computational Linguistics.

Ralph Weischedel, Martha Palmer, Mitchell Marcus, Eduard Hovy, Sameer Pradhan, Lance Ramshaw, Nianwen Xue, Ann Taylor, Jeff Kaufman, Michelle Franchini, et al. 2013. Ontonotes release 5.0 ldc2013t19. Linguistic Data Consortium, Philadelphia, PA, 23.

Wei Wu, Fei Wang, Arianna Yuan, Fei Wu, and Jiwei Li. 2020. CorefQA: Coreference resolution as query-based span prediction. In Proceedings of the 58th Annual Meeting of the Association for Computational Linguistics, pages 6953-6963, Online. Association for Computational Linguistics.
Steve Young. 2002. Talking to machines (statistically speaking). In Seventh International Conference on Spoken Language Processing.

Xiaoxue Zang, Abhinav Rastogi, Srinivas Sunkara, Raghav Gupta, Jianguo Zhang, and Jindong Chen. 2020. MultiWOZ 2.2 : A dialogue dataset with additional annotation corrections and state tracking baselines. In Proceedings of the 2nd Workshop on Natural Language Processing for Conversational AI, pages 109-117, Online. Association for Computational Linguistics. 


\section{A Details of Question Generation}

The part-of-speech tagset is based on the Universal Dependencies scheme ${ }^{4}$. The named entity labels are based on OntoNotes 5.0 (Weischedel et al., 2013). In Table 6, we describe the set of rules for the selection of WH_word.

\section{B Inference Constraints}

At inference time, predicting the slot filler spans is more complex - for each slot type, as there can be several or no spans to be extracted. After the output layer, we have the probability of each token $x_{i}$ being the start $\left(P_{\mathrm{s}}(i)\right)$ and end $\left(P_{\mathrm{e}}(i)\right)$ of the span.

We harvest all the valid candidate spans for each slot type with the following heuristics:

1. Enumerate all possible combinations of start offset (start) and end offset (end) of the spans $\left(\frac{M(M-1)}{2}\right.$ candidates in total);

2. Eliminate the spans not satisfying the constraints: (1) start and end token must be within the utterance; (2) the length of the span should be shorter than a maximum length constraint; (3) spans should have a larger probability than the probability of "no answer" (which is represented with [CLS] token), namely,

$$
P_{\mathrm{s}}(\text { start })>P_{\mathrm{s}}([\mathrm{CLS}]), P_{\mathrm{e}}(\text { end })>P_{\mathrm{e}}([\mathrm{CLS}])
$$

\section{Further Analysis and Discussions}

We conduct further analysis to understand how and why the models are effective.

\section{C.1 Impact of question generation strategy and pretraining}

Table 7 shows full ablation results.

\section{C.2 Analysis on Seen versus Unseen Slots}

To understand the transferring capability of our models, we further split the SNIPS test for each target domain into "seen" and "unseen" slots. An example is categorized into "unseen" as long as there is an unseen slot (i.e., the slot does not exist in the remaining six source domains in its gold annotation.) Otherwise, it counts as "seen". A full list of unseen slots for each target domain can be found in the Appendix.

\footnotetext{
${ }^{4}$ universaldependencies.org/u/pos/
}

As is shown in Table 5, we can see that (1) both ZSBT baseline and our models perform better on the "seen" slots than the "unseen" ones - the numbers substantially drop on the "unseen" slots. This proves that transferring from the source domains to the unseen slots in the target domain is a hard problem. (2) On the portion of examples with "seen" slots, our best model outperforms ZSBT with around a $2 \%$ margin. (3) On the "unseen" portion of examples, the margin is larger - our QASF and pretraining step help improve the performance more (over 4\%). The second and third observation together demonstrates that the questioning strategy help improve the model's capability of transferring between related but not exactly same slot types (e.g., "object_name" and "entity_name").

\begin{tabular}{l|cc}
\hline & Seen & Unseen \\
\hline ZSBT & 54.75 & 37.41 \\
QASF & 56.79 & 39.99 \\
w/ pretraining & 56.23 & 41.73 \\
\hline
\end{tabular}

Table 5: Averaged F-1 scores over all target domains of SNIPS dataset (for "unseen" and seen "slots").

\section{Hyper-parameters and Training Details}

We use the uncased version of the BERT-base (Devlin et al., 2019) model for QA finetuning and pretraining. The model is fine-tuned for 5 epochs with a starting learning rate of $3 e-5$ on the SNIPS dataset. The model is pretrained for 5 epochs with a starting learning rate of $5 \mathrm{e}-7$ on the synthetic QA dataset. Our implementations are based on https://github.com/google-research/bert/ blob/master/run_squad.py 


\begin{tabular}{|c|c|c|}
\hline WH_word & Conditions & Answer Examples \\
\hline How long & $\begin{array}{l}\text { The answer phrase is modified by a cardinal number (CARD INAL) } \\
\text { or quantifier phrase (QUANT I TY) whose object is a temporal unit, } \\
\text { as is defined in (Pan et al., 2011), i.e., second/minute/hour/day/week/month/year/decade/century. }\end{array}$ & 2 nights \\
\hline How many & $\begin{array}{l}\text { The answer phrase is modified by a cardinal number (CARDINAL) } \\
\text { or quantifier phrase (QUANTITY) and the object is not a temporal unit. }\end{array}$ & 2 stars, 3 tickets \\
\hline How & adjective $\mathrm{ADJ}$ & moderate, expensive \\
\hline When & The answer phrase's head word is tagged DATE or TIME & 1:30 PM, 1999 \\
\hline Who & $\begin{array}{l}\text { The answer phrase's head word is tagged PERSON or } \\
\text { is a personal pronoun PRON (I, he, herself, them, etc.) }\end{array}$ & mother, Dr. Williams \\
\hline Where & $\begin{array}{l}\text { The answer phrase is a prepositional phrase whose object is tagged } \\
\text { GPE or LOC, whose preposition is one of the following: on, in, at, over, to }\end{array}$ & $\begin{array}{l}\text { amc theaters, } \\
\text { fort point san francisco, } \\
\text { east, west, ... }\end{array}$ \\
\hline Which & The answer phrase is a determiner DT (this, that) or an ordinal ORDINAL & $\begin{array}{l}\text { this, first, } \\
\text { current, last }\end{array}$ \\
\hline What & all other cases & \\
\hline
\end{tabular}

Table 6: Strategy for Generating the WH_word (question phrase).

\begin{tabular}{l|ccccccc|c}
\hline & ATP & BR & GW & PM & RB & SCW & FSE & Average F1 \\
\hline \multicolumn{8}{c}{ Q/o pretraining } \\
QASF & 59.29 & 43.13 & 59.02 & 33.62 & 33.34 & 59.90 & 22.83 & 44.45 \\
w/o question & 55.30 & 46.71 & 53.06 & 35.79 & 25.28 & 59.77 & 17.85 & 41.97 \\
\hline \multicolumn{8}{c}{ w/ pretraining } \\
QASF & 57.57 & 48.75 & 61.27 & 38.54 & 36.51 & 60.82 & 27.72 & 47.31 \\
w/o question & 56.11 & 42.42 & 55.70 & 33.07 & 33.13 & 60.03 & 21.20 & 43.09 \\
\hline
\end{tabular}

Table 7: Full ablation analysis on SNIPS dataset. 


\section{E Schema of SNIPS dataset}

\begin{tabular}{|c|c|c|}
\hline Domain & All Slots & Unseen Slots \\
\hline AddToPlaylist (ATP) & $\begin{array}{l}\text { entity_name } \\
\text { playlist_owner } \\
\text { playlist } \\
\text { artist } \\
\text { music_item }\end{array}$ & $\begin{array}{l}\text { entity_name } \\
\text { playlist_owner }\end{array}$ \\
\hline BookRestaurant (BR) & $\begin{array}{l}\text { restaurant_type } \\
\text { served_dish } \\
\text { restaurant_name } \\
\text { party_size_description } \\
\text { cuisine } \\
\text { party_size_number } \\
\text { timerange } \\
\text { facility } \\
\text { poi } \\
\text { state } \\
\text { city } \\
\text { country } \\
\text { sort } \\
\text { spatial_relation }\end{array}$ & $\begin{array}{l}\text { restaurant_type } \\
\text { served_dish } \\
\text { restaurant_name } \\
\text { party_size_description } \\
\text { cuisine } \\
\text { party_size_number } \\
\text { timerange } \\
\text { facility } \\
\text { poi }\end{array}$ \\
\hline GetWeather (GW) & $\begin{array}{l}\text { timerange } \\
\text { current_location } \\
\text { condition_description } \\
\text { geographic_poi } \\
\text { condition_temperature } \\
\text { country } \\
\text { state } \\
\text { city } \\
\text { spatial_relation }\end{array}$ & $\begin{array}{l}\text { timerange } \\
\text { current_location } \\
\text { condition_description } \\
\text { geographic_poi } \\
\text { condition_temperature }\end{array}$ \\
\hline PlayMusic (PM) & $\begin{array}{l}\text { year } \\
\text { genre } \\
\text { service } \\
\text { album } \\
\text { track } \\
\text { sort } \\
\text { music_item } \\
\text { artist } \\
\text { playlist }\end{array}$ & $\begin{array}{l}\text { year } \\
\text { genre } \\
\text { service } \\
\text { album } \\
\text { track }\end{array}$ \\
\hline RateBook (RB) & $\begin{array}{l}\text { object_select } \\
\text { rating_value } \\
\text { best_rating } \\
\text { rating_unit } \\
\text { object_part_of_series_type } \\
\text { object_type } \\
\text { object_name }\end{array}$ & $\begin{array}{l}\text { object_select } \\
\text { rating_value } \\
\text { best_rating } \\
\text { rating_unit } \\
\text { object_part_of_series_type }\end{array}$ \\
\hline SearchCreativeWork (SCW) & $\begin{array}{l}\text { object_type } \\
\text { object_name }\end{array}$ & - \\
\hline FindScreeningEvent (FSE) & $\begin{array}{l}\text { object_location_type } \\
\text { movie_name } \\
\text { movie_type } \\
\text { timerange } \\
\text { location_name } \\
\text { object_type } \\
\text { spatial_relation }\end{array}$ & $\begin{array}{l}\text { object_location_type } \\
\text { movie_name } \\
\text { movie_type } \\
\text { timerange } \\
\text { location_name }\end{array}$ \\
\hline
\end{tabular}

Table 8: Schema of SNIPS dataset 


\section{F Question Templates for SNIPS}

\begin{tabular}{|c|c|c|c|}
\hline Domain & Slot & Slot Name & Natural Question \\
\hline AddToPlaylist & music_item & music item & what's the music item? \\
\hline AddToPlaylist & playlist_owner & owner & who's the owner? \\
\hline AddToPlaylist & entity_name & entity name & what's the entity name? \\
\hline AddToPlaylist & playlist & playlist & what's the playlist? \\
\hline AddToPlaylist & artist & artist & who's the artist? \\
\hline BookRestaurant & city & city & what's the city? \\
\hline BookRestaurant & facility & facility & what's the facility? \\
\hline BookRestaurant & timeRange & time range & when's the time range? \\
\hline BookRestaurant & restaurant_name & restaurant name & what's the name? \\
\hline BookRestaurant & country & country & what's the country? \\
\hline BookRestaurant & cuisine & cuisine & what's the cuisine? \\
\hline BookRestaurant & restaurant_type & restaurant type & what's the restaurant type? \\
\hline BookRestaurant & served_dish & served dish & what's the served dish? \\
\hline BookRestaurant & party_size_number & number & how many people? \\
\hline BookRestaurant & poi & position & where's the location? \\
\hline BookRestaurant & sort & type & what's the type? \\
\hline BookRestaurant & spatial_relation & spatial relation & what's the spatial relation? \\
\hline BookRestaurant & state & location & what's the state? \\
\hline BookRestaurant & party_size_description & person & who are the persons? \\
\hline GetWeather & city & city & what's the city? \\
\hline GetWeather & state & location & what's the state? \\
\hline GetWeather & timeRange & time range & when's the time range? \\
\hline GetWeather & current_location & current location & what's the current location? \\
\hline GetWeather & country & country & what's the country? \\
\hline GetWeather & spatial_relation & spatial relation & what's the spatial relation? \\
\hline GetWeather & geographic_poi & geographic position & where's the location? \\
\hline GetWeather & condition_temperature & temperature & how is the temperature? \\
\hline GetWeather & condition_description & weather & how is the weather? \\
\hline PlayMusic & genre & genre & what's the genre? \\
\hline PlayMusic & music_item & music item & what's the music item? \\
\hline PlayMusic & service & service & what's the service \\
\hline PlayMusic & year & year & when's the year? \\
\hline PlayMusic & playlist & playlist & what's the playlist? \\
\hline PlayMusic & album & album & what's the album? \\
\hline PlayMusic & sort & type & what's the type? \\
\hline PlayMusic & track & track & what's the track? \\
\hline PlayMusic & artist & artist & who's the artist? \\
\hline RateBook & object_part_of_series_type & series & what's the series? \\
\hline RateBook & object_select & this current & which to select? \\
\hline RateBook & rating_value & rating value & how many rating value? \\
\hline RateBook & object_name & object name & what's the object name? \\
\hline RateBook & object_type & object type & what's the object type? \\
\hline RateBook & rating_unit & rating unit & what's the rating unit? \\
\hline RateBook & best_rating & best rating & how many rating points in total? \\
\hline SearchCreativeWork & object_name & object name & what's the object name? \\
\hline SearchCreativeWork & object_type & object type & what's the object type? \\
\hline SearchScreeningEvent & timeRange & time range & when's the time range? \\
\hline SearchScreeningEvent & movie_type & movie type & what's the movie type? \\
\hline SearchScreeningEvent & object_location_type & location type & what's the location type? \\
\hline SearchScreeningEvent & object_type & object type & what's the object type? \\
\hline SearchScreeningEvent & location_name & location name & where's the location name? \\
\hline SearchScreeningEvent & spatial_relation & spatial relation & what's the spatial relation? \\
\hline SearchScreeningEvent & movie_name & movie name & what's the movie name? \\
\hline
\end{tabular}

Table 9: Question Templates for SNIPS 